\title{
Enfermedad tromboembólica venosa en el embarazo
}

\author{
Venous thromboembolic disease in pregnancy
}

Dra. Paola Silva-Reyna*

RESUMEN. La enfermedad tromboembólica venosa como trombosis venosa profunda y tromboembolismo pulmonar, son causa importante de mortalidad materna, además se han considerado un problema de salud pública en diferentes países. La trombosis venosa profunda y el tromboembolismo pulmonar son considerados parte de una misma enfermedad con diversos síntomas clínicos que afectan al paciente. Algunas cifras epidemiológicas de la enfermedad tromboembólica venosa son que su incidencia se encuentra en torno a 167 por cada 1,000,000 personas por año, en mujeres embarazadas se presenta con una incidencia de 0.5-2.2 por 1,000 embarazos además que es de elevada mortalidad, se ha incrementado en los últimos diez años y en sus factores de riesgo podemos mencionar: trombofilia, tabaquismo, raza, hipertensión, hemorragia obstétrica, transfusiones, obesidad, cesárea principalmente. El abordaje terapéutico dependerá de la expresión clínica de la enfermedad tromboembólica venosa. Por lo que esta revisión incluye datos sobre la epidemiología, patogénesis, inflamación, factores de riesgo relacionados a la enfermedad tromboembólica venosa en el embarazo, la identificación de factores de riesgo no sólo son fundamentales para el diagnóstico, sino que también son importantes para prevenir y tratar la enfermedad.

ABSTRACT. Venous thromboembolic disease such as deep venous thrombosis and pulmonary thromboembolism are important causes of maternal mortality and has also been considered a public health problem in different countries. Deep venous thrombosis and pulmonary thromboembolism are considered part of the same disease with various clinical symptoms that affect the patient. Some epidemiological numbers of the venous thromboembolic disease are that its incidence is around 167 for every 1,000,000 people per year, in pregnant women it appears with an incidence of 0.5-2.2 per 1,000 pregnancies besides that it is of high mortality, it has increased in the last ten years and in their risk factors we can mention: thrombophilia, smoking, race, hypertension, obstetric hemorrhage, transfusions, obesity, cesarean section mainly. The therapeutic approach will depend on the clinical expression of venous thromboembolic disease. So this review includes data on the epidemiology, pathogenesis, inflammation, risk factors related to venous thromboembolic disease in pregnancy, the identification of risk factors are not only essential for diagnosis, but are also important to prevent and treat the disease.

Abreviaturas:

ETV $=$ Enfermedad tromboembólica venosa.

$\mathrm{TP}=$ Tromboembolismo pulmonar.

TVP = Trombosis venosa profunda.

\section{INTRODUCCIÓN}

La enfermedad tromboembólica venosa (ETV) como trombosis venosa profunda (TVP) y tromboembolismo pulmonar (TP), son importantes causas de mortalidad materna, además se han considerado un problema de salud pública $^{(1)}$. La ETV es una enfermedad que incluye las interacciones entre las predisposiciones adquiridas o heredadas a la trombosis y los factores de riesgo de ETV ${ }^{(2)}$. Durante la gestación, la TVP es más frecuente en las extremidades inferiores y es responsable del 75 al $80 \%$ de los casos de $\mathrm{ETV}^{(3)}$. En un estudio caso-control realizado en mujeres

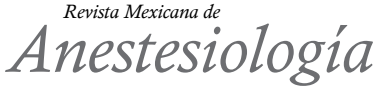

Enero-Marzo 2020

Vol. 43. No. 1. pp 41-47

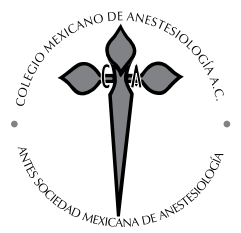

*Médico residente de tercer año de la Especialidad de Anestesiología de la Facultad Mexicana de Medicina Universidad La Salle con sede en el Hospital Ángeles Metropolitano.

\section{Palabras clave:}

Enfermedad tromboembólica venosa, embarazo, tromboprofilaxis.

Keywords:

Venous thromboembolic disease, pregnancy, thromboprophylaxis.

Solicitud de sobretiros:

Dra. Paola Silva-Reyna

Tlacotalpan Núm. 59,

Col. Roma Sur,

06760, Del. Cuauhtémoc,

Ciudad de México.

Tel: 5514888383

E-mail:

paola@anestesiologa.com.mx

Recibido para publicación:

24-11-2017

Aceptado para publicación:

14-09-2018 embarazadas, se observó que la TVP se presentó predominantemente en el miembro inferior izquierdo $(78.4 \%)^{(4)}$. Este fenómeno se puede atribuir a un aumento de la estasis venosa en la pierna izquierda en relación a la compresión de la vena ilíaca común izquierda por la arteria ilíaca común derecha contra la quinta vértebra lumbar, causada por el útero grávido ${ }^{(3)}$.

Asimismo, la ETV en el embarazo constituye una causa importante de morbimortalidad materna en los países desarrollados, se presenta con una incidencia de $0.5-2.2$ por 1,000 embarazos ${ }^{(5)}$. La incidencia de ETV durante la gestación fue de 85/100,000 embarazos en Inglaterra ${ }^{(6)}$ y 199 por 100,000 
años-mujer en Minnesota Estados Unidos ${ }^{(7)}$. Galambosi PJ et al., reportaron que la incidencia de eventos tromboembólicos venosos fueron más altos durante la primera semana postparto, por lo que el riesgo se mantiene en los primeros 180 días aunque disminuye conforme pasa el tiempo ${ }^{(8)}$. En México la ETV se ha estimado en más de 500 mil casos por año ${ }^{(9)}$. Asimismo, la incidencia de TVP va de un caso por 10,000 adultos jóvenes. En adultos entre 65 a 69 años de edad la incidencia de TVP fue de 1.8 casos por 1,000 habitantes por año y aumenta a 3.1 casos por 1,000 habitantes por año entre 85 y 89 años de edad ${ }^{(10)}$.

De la misma forma, diariamente fallecen en el mundo aproximadamente 830 mujeres por complicaciones relacionadas con el embarazo o el parto ${ }^{(11)}$. Las principales causas de mortalidad materna reportadas en países desarrollados han sido la enfermedad hipertensiva (16.1\%), tromboembolia pulmonar (14.9\%) y la hemorragia $(13.4 \%)$ y para América Latina se ha reportado la enfermedad hipertensiva $(25.7 \%)$, la hemorragia $(20.8 \%)$ y el aborto $(12.0 \%)^{(12)}$. Existen estudios donde mencionan que las mujeres embarazadas tienen mayor probabilidad de desarrollar enfermedad tromboembólica en comparación con las mujeres que no se encuentran embarazadas ${ }^{(13,14)}$. En México entre 1991-2005 la mortalidad materna hospitalaria registró un descenso de $40.4 \%$, ya que la tasa pasó de 45.3 a 27 por 100,000 nacidos vivos, asimismo el número de muertes maternas disminuyó ya que la tasa se redujo de 34.6 a 25.9 por 100,000 nacidos vivos ${ }^{(15)}$.

\section{PATOGÉNESIS}

Fisiológicamente el embarazo y el puerperio presentan una serie de cambios que aumentan el riesgo de desarrollar TVP, ya que durante el mismo coinciden los tres criterios propuestos por Virchow, que son: 1) Hipercoagulabilidad, la gestación provoca un aumento de los factores de coagulación VII, VIII y X por influencia del estriol placentario, también existe aumento de los niveles de fibrinógeno y de los inhibidores del activador del plasminógeno de tipo I y II, y hay una síntesis reducida de proteína $S^{(16)}$. 2) Estasis Venosa, en extremidades inferiores se produce durante el embarazo debido a dos factores: los cambios asociados con el embarazo en la capacitancia venosa y la comprensión de las grandes venas por el útero grávido. Aunque el volumen de sangre y el retorno venoso total están aumentados en el embarazo, la velocidad de flujo lineal en las venas de las extremidades inferiores se reduce debido a la dilatación inducida hormonalmente, lo que lleva a estasia e incompetencia valvular. Estos cambios tempranos son amplificados por la comprensión de la vena cava inferior y de la vena ilíaca por el útero grávido. 3) Daño endotelial, el parto produce lesión vascular y cambios en la superficie uteroplacentaria, lo que probablemente contribuye al aumento del riesgo de ETV en el postparto inmediato. La cesárea puede aumentar aun más las lesiones de la íntima vascular y amplificar este fenómeno ${ }^{(17)}$.

\section{INFLAMACIÓN}

Se conoce que la inflamación desempeña un papel importante en los procesos inflamatorios de varias enfermedades, además que son factores de riesgo para TVP. Las células inflamatorias como neutrófilos, linfocitos, monocitos, macrófagos, citocinas proinflamatorias y plaquetas activadas intervienen en este proceso ${ }^{(18)}$.

Los neutrófilos son el primer tipo de célula inmune que responde y llega al sitio de la infección, en el proceso inflamatorio se lleva a cabo una producción y liberación de estructuras en forma de redes, llamadas trampas extracelulares de neutrófilos (TEN), compuestas por ADN, histonas y otras proteínas antimicrobianas, en respuesta a la presencia de microorganismos patógenos ${ }^{(19)}$. Fuchs TA et al., observaron que las TEN estimulan la adhesión plaquetaria y la coagulación, además que las TEN interactúan con el endotelio, las plaquetas y los factores de coagulación y pueden influir en la trombólisis ${ }^{(20)}$. Además las TEN podrían tener una interacción principal entre la inflamación y la trombosis debido a que las TEN proporcionan un andamio que brinda estabilidad a los trombos.

En un estudio realizado en 2010 por Fuchs TA et al., se demostró que las TEN no sólo aportan un andamio sino también estímulos que permiten la activación, adhesión y agregación de plaquetas, adhesión de glóbulos rojos y de proteínas plasmáticas implicadas en la estabilidad del trombo, como son la fibronectina, el fibrinógeno y el factor de Von Willebrand ${ }^{(21)}$.

\section{FACTORES DE RIESGO}

Durante la gestación y el parto diversos factores podrían contribuir a incrementar el riesgo de la formación de trombosis, existen factores que predisponen a la ETV como son: el incremento de la edad materna, obesidad, parto por cesárea, inmovilización por amenaza de aborto, amenaza de parto prematuro, deshidratación por hiperémesis, enfermedades cardíacas, historia de trombosis ${ }^{(22,23)}$ y trombofilia; en el cuadro I se describen otros factores de riesgo que pueden estar relacionados a la ETV ${ }^{(24)}$.

\section{TROMBOFILIA}

Las trombofilias se relacionan con la morbilidad y mortalidad materno-fetal, que se encuentran asociadas a eventos tromboembólicos venosos y arteriales en el período grávido 
Tabla 1: Factores de riesgo para enfermedad tromboembólica venosa (ETV) en el embarazo.

\begin{tabular}{|c|c|c|}
\hline Factores de riesgo para ETV & ${ }^{*}$ RM Ajustado & IC $95 \%$ \\
\hline Previo ETV & 24.8 & $17.1-36.0$ \\
\hline Inmovilidad & 7.7 & $3.2-19$ \\
\hline si combinada con IMC $\geq 25$ & 62 & \\
\hline $\mathrm{IMC}>30$ & 5.3 & $2.1-13.5$ \\
\hline Fumar & 2.7 & $1.5-4.9$ \\
\hline Aumento de peso > 21 kg (vs 7- $21 \mathrm{~kg}$ ) & 1.6 & $1.1-2.6$ \\
\hline Parto $>1$ & 1.5 & $1.1-1.9$ \\
\hline Edad $>35 y$ & 1.3 & $1.0-1.7$ \\
\hline Preeclampsia & 3.1 & $1.8-5.3$ \\
\hline Preeclampsia con restricción de crecimiento fetal & 5.8 & $2.1-16$ \\
\hline Técnicas de reproducción asistidas & 4.3 & $2.0-9.4$ \\
\hline Embarazo gemelar & 2.6 & $1.1-6.2$ \\
\hline Hemorragia ante-parto & 2.3 & $1.8-2.8$ \\
\hline Hemorragia post-parto & 4.1 & $2.3-7.3$ \\
\hline Sección de cesárea & 3.6 & $3.0-4.3$ \\
\hline $\begin{array}{l}\text { Condición médica como lupus eritematoso, enfermedad del corazón, anemia, infección activa, } 0 \\
\text { venas varicosas }\end{array}$ & $2.0-8.7$ & \\
\hline Transfusión de sangre & 7.6 & $6.2-9.4$ \\
\hline
\end{tabular}

*RM= Razón de Momios. Tomado de James AH. Arterioscler Thromb Vasc Biol. 2009;29(3):326-31.

\section{Tabla 2: Riesgo de enfermedad tromboembólica venosa} (ETV) conferido por tipo de trombofilia.

\section{Trombofilia}

Factor V Leiden- homocigosidad

Factor $\mathrm{V}$ Leiden- heterocigosidad

Mutación del gen de protrombina-homocigo-

sidad

Mutación del gen de protrombina-heteroci-

gosidad

Deficiencia de proteína $\mathrm{C}$

Deficiencia de Proteína S

Deficiencia de antitrombina

*RM= Razón de Momios. Tomado de James AH. Arterioscler Thromb Vasc Biol. 2009;29(3):326-31.

puerperal, asimismo diferentes investigaciones hacen mención que la trombofilia aumenta el riesgo de tromboembolismo venoso y también la trombofilia se relaciona a eventos adversos en el embarazo como son la pérdida del embarazo, la preeclampsia, el desprendimiento de la placenta y la restricción del crecimiento intrauterino ${ }^{(25)}$.

Un estudio de cohorte en Canadá que incluyó mujeres embarazadas, encontró una incidencia de TVP de 12.1, asimismo observó que las mujeres embarazadas con TVP tuvieron mayor probabilidad de presentar trombofilia (RM $=15.4$; IC95\% 10.8-22.0) $)^{(26)}$, en otro estudio James AH et al. reportaron una $\mathrm{RM}=51.8$ (IC95\% 38.7-69.2) para trom- bofilia ${ }^{(33)}$. De igual manera otras investigaciones mencionan que la trombofilia se encuentra presente entre el 20 y $50 \%$ de las mujeres que experimentan ETV durante el embarazo y el período postparto ${ }^{(27,28)}$. Asimismo existe asociación entre ETV y efectos adversos en el embarazo, en una revisión sistemática llevada a cabo por Robertson L et al., encontraron que las mujeres con trombofilia tienen mayor probabilidad de desarrollar ETV y complicaciones durante el embarazo, además propusieron el riesgo absoluto de ETV asignado por tipo de la trombofilia que se presenta en el cuadro $\mathrm{II}^{(29)}$.

\section{OBESIDAD}

La presencia de obesidad es otro factor de riesgo importante de tromboembolismo venoso en el embarazo y el puerperio y el riesgo aumenta a medida que incrementa el índice de masa corporal (IMC) $)^{(30,31)}$. En un estudio en Dinamarca se encontró que la obesidad (IMC $>30 \mathrm{~kg} / \mathrm{m}^{2}$ ) fue asociada con el riesgo de incrementar ETV durante el embarazo y el puerperio $\left(\mathrm{RM}=5.3\right.$; IC95\% 2.1-13.5) ${ }^{(32)}$. De igual modo James AH et al., encontraron que la presencia de obesidad aumenta el riesgo de ETV en el embarazo ( $\mathrm{RM}=4.4$; IC95\% $3.4-5.7)^{(33)}$.

\section{OTROS FACTORES DE RIESGO}

Por otra parte, otras condiciones médicas que aumentan el riesgo de ETV son enfermedades del corazón, lupus eritematoso sistémico, anemia, diabetes, hipertensión arterial inducida por 


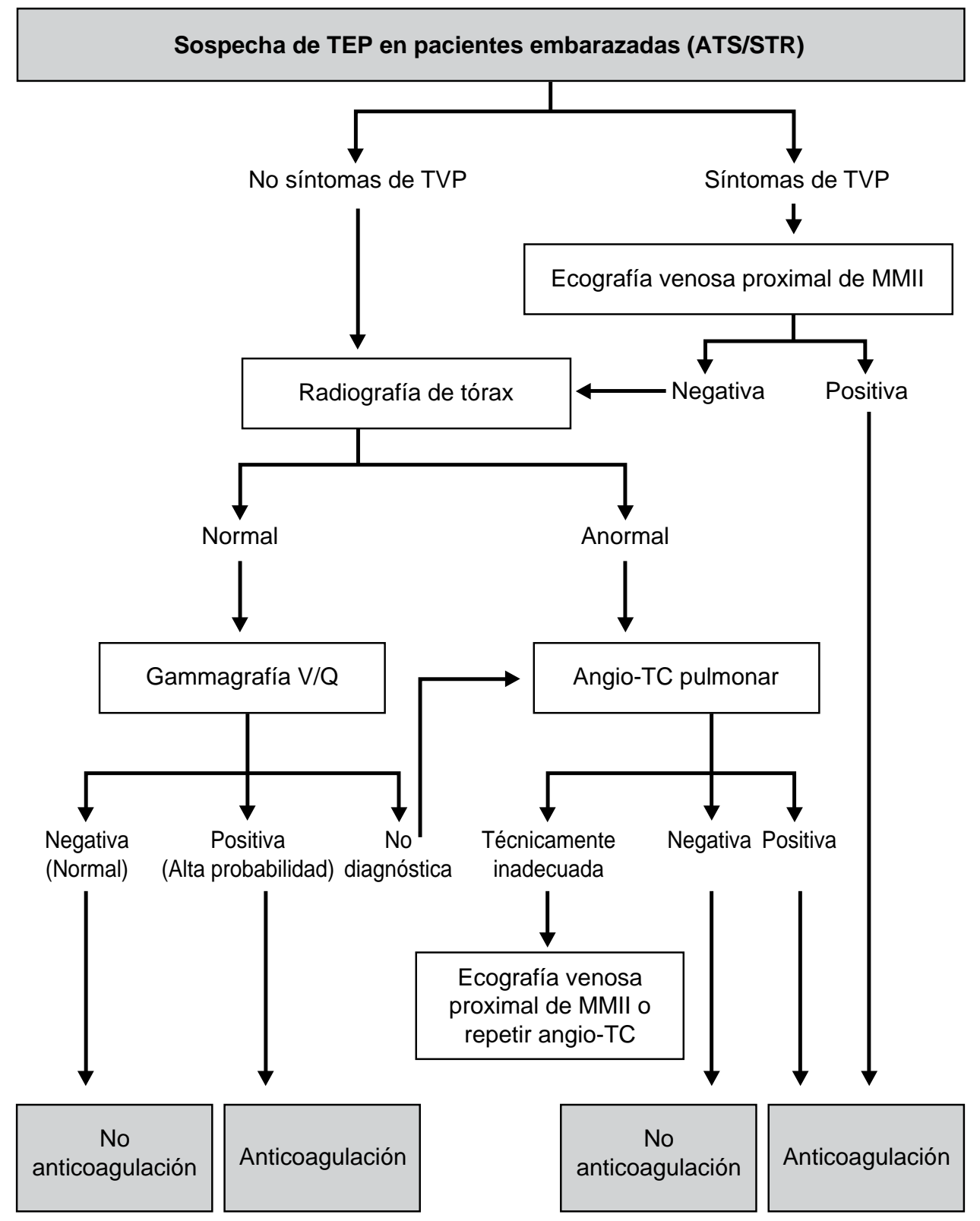

Figura 1:

Algoritmo de la guía clínica de la American Thoracic Society (ATS) y de la Society of Thoracic Radiology (STR). MMIl: miembros inferiores; TEP: tromboembolia pulmonar; TVP: trombosis venosa profunda.

el embarazo o preeclampsia ${ }^{(34)}$, tabaquismo ${ }^{(23)}$, multiparidad, ETV previa, infecciones, cesárea, hemorragia obstétrica, restricción del crecimiento intrauterino, muerte fetal, hiperémesis y hospitalización ${ }^{(35)}$. Abbasi $\mathrm{N}$ et al., encontraron que la edad materna mayor a 25 años, tabaquismo, trombofilia, enfermedad cardiovascular, hipertensión, obesidad, hemorragia postparto y transfusión sanguínea fueron asociados a $\mathrm{ETV}^{(36)}$.

Durante el período entre 2000 y 2001 de 9,058,162 ingresos por embarazo y 73,834 admisiones postparto, se identificaron factores de riesgo asociados a ETV que incluyen: lupus (OR $=8.7$; IC95\% 5.8-13.0), diabetes $(\mathrm{OR}=2.0 ; \mathrm{IC} 95 \%$ 1.4-2.7 $)$, tabaquismo $(\mathrm{OR}=1.7$; IC95\% 1.4-2.1), hipertensión $(\mathrm{OR}=$
1.8; IC95\% 1.4-2.3) y enfermedades del corazón $(\mathrm{OR}=7.1$; IC95\% 6.2-8.3) ${ }^{(33)}$.

\section{DIAGNÓSTICO}

En la actualidad no existe una escala clínica validada para mujeres embarazadas para el diagnóstico de $\mathrm{TP}^{(37)}$. En un estudio retrospectivo se obtuvo alta sensibilidad y especificidad de los criterios de la escala de Wells modificados en gestantes, pero se requiere mayor investigación ${ }^{(38)}$. También se ha propuesto la regla LEFt de predicción de TVP, que combina síntomas en pierna izquierda (left, L), diferencia de la pantorrilla $>2 \mathrm{~cm}$ res- 


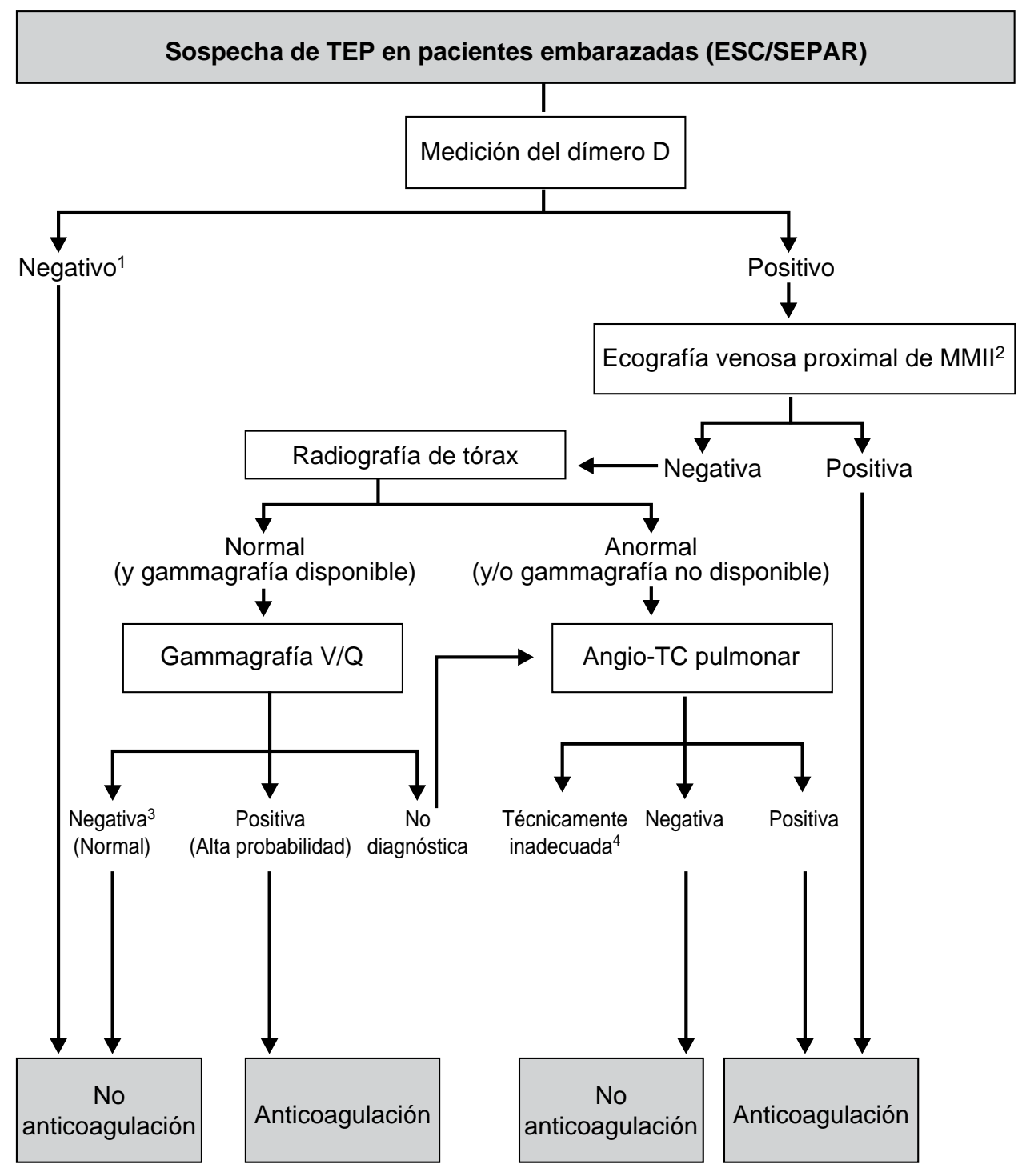

Figura 2:

Algoritmo basado en las recomendaciones de la European Society of Cardiology (ESC) de 2014 y la normativa de la Sociedad Española de Neumología y Cirugía Torácica (SEPAR) de 2013. DD: dímero D; MMII: miembros inferiores; TEP: tromboembolia pulmonar. ${ }^{1}$ La guía de la ESC emplea el dímero $D$ (IIbC), y la normativa SEPAR directamente realiza ecografía de MMII (no hace mención al dímero D).

${ }^{2}$ La ecografía venosa de MMIl es la prueba de imagen de primera línea independientemente de la existencia o no de síntomas en piernas.

${ }^{3}$ La ecografía venosa de MMII es la prueba de imagen de primera línea independientemente de la existencia o no de síntomas en piernas. pecto a la contralateral (edema, E) y presentación en el primer trimestre (first trimester, Ft). Si no se cumple ninguno de los criterios LEFt la probabilidad de TVP es muy baja.

El dímero D se eleva fisiológicamente en el curso del embarazo y supera con frecuencia el valor de corte diagnóstico, con una menor especificidad de la prueba, por lo que es controvertido. Por lo contrario otros autores obtuvieron un valor predictivo negativo para TVP del $100 \%$ en gestantes en primer y segundo trimestre, con una prueba de aglutinación altamente específica, aunque faltan estudios prospectivos amplios que lo validen ${ }^{(39)}$.

\section{RECOMENDACIONES DE LAS GUIIAS CLÍNICAS}

En 2011, la American Thoracic Society (ATS) y la Society of Thoracic Radiology (STR) elaboraron conjuntamente una guía de práctica clínica para evaluar la sospecha de TP en el embarazo (Figura 1).

No recomiendan el uso del Dímero D; indican una radiografía de tórax a toda embarazada con sospecha de TP y una ecografía venosa de compresión de MMI sólo en caso de síntomas de TVP. Sin embargo en la guía de la ESC de 2014 y en la normativa de la SEPAR 2013 (Figura 2) la prueba de imagen de primera línea en el embarazo es la ecografía venosa de compresión de MMII, incluso en ausencia de síntomas de las piernas. Por otro lado, todas las guías coinciden en emplear gammagrafía si la radiografía de tórax es normal y no hay TVP, para evitar radiación mamaria. La angio-TC debe considerarse si la radiografía es anormal, no se dispone fácilmente de gammagrafía o no es diagnosticada y se decide seguir estudiando ${ }^{(40)}$. 


\section{TROMBOPROFILAXIS}

En toda paciente gestante se debe realizar al inicio de la gestación una evaluación clínica del riesgo trombótico. Actualmente se han realizado investigaciones sobre ETV durante el embarazo y se han propuesto recomendaciones sobre la prevención y el tratamiento farmacológico ${ }^{(41,42)}$. Para el tratamiento farmacológico se usan diferentes fármacos anticoagulantes, pero los de mayor recomendación durante la gestación son la heparina de bajo peso molecular (HBPM) y heparina no fraccionada (HNF). Middeldorp S. reportó que la HBPM en dosis terapéuticas es el tratamiento de elección durante la gestación ${ }^{(43)}$.

En una revisión sistemática se incluyeron 64 estudios con mujeres embarazadas donde se evaluó la seguridad y eficacia de la HBPM para la tromboprofilaxis y el tratamiento de ETV en el embarazo, dentro de los principales resultados se encontró que en 15 estudios, el tratamiento para ETV fue el uso de la HBPM, estas mujeres fueron tratadas con enoxaparina, dalteparina y nadroparina. La HBPM se administró dos veces al día en $153 \operatorname{casos}^{(44)}$. Asimismo en la misma revisión sistemática se reportó el uso de la HBPM en dosis tromboprofilácticas, por lo que se concluye que el uso de la HBPM durante el embarazo es seguro y efectivo para tratar y prevenir la trombosis en el embarazo ${ }^{(44)}$.

Por otra parte, en una revisión sistemática donde el objetivo fue determinar los efectos de la tromboprofilaxis en mujeres embarazadas o que hubieran tenido un parto reciente con mayor probabilidad de ETV, se concluyó que no hay estudios aleatorizados suficientes sobre los cuales basar las recomendaciones para la tromboprofilaxis durante el embarazo y el período postnatal temprano ${ }^{(45,46)}$. Asimismo en un estudio donde se evaluó el riesgo de ETV y las prácticas de tromboprofilaxis se reportó que del total de los pacientes que presentaban algún riesgo, sólo el 22.2\% siguieron las medidas tromboprofilácticas adecuadas, asimismo el 78.0\% quedó desprotegido debido a que en el $58.8 \%$ las medidas fueron inadecuadas y en el $18.8 \%$ no hubo medidas profilácticas, por lo que es indispensable la implementación de las recomendaciones para tromboprofilaxis e iniciar estrategias que involucren al personal médico para disminuir la ETV ${ }^{(47)}$.

\section{RECOMENDACIONES}

Existen guías que resumen información disponible y de calidad con el objetivo de proporcionar enfoques prácticos para el diagnóstico, prevención y tratamiento de la ETV en el embarazo, una de ellas es la propuesta por Chan WS et al., 2014(48), la cual proporciona un enfoque basado en la evidencia reciente de la información para el diagnóstico, tratamiento y tromboprofilaxis de la ETV en el embarazo y el postparto. Dentro de las recomendaciones importantes podemos mencionar el uso de la HBPM que es el agente farmacológico preferido sobre la heparina no fraccionada para el tratamiento de la tromboembolia venosa en el embarazo. La HBPM puede ser administrada una o dos veces al día dependiendo del agente seleccionado. Los recuentos basales de plaquetas deben tomarse y repetirse una semana después para detectar la trombocitopenia inducida por heparina ${ }^{(48)}$.

Asimismo existe otra guía que proporciona orientación clínica práctica sobre la prevención y tratamiento de la ETV asociado a la gestación de acuerdo a la información actual disponible. Algunos puntos importantes que se deben considerar es que la HBPM es el anticoagulante seguro para las mujeres embarazadas con ETV aguda ${ }^{(49)}$. Tratamiento extendido: la terapia anticoagulante para el tratamiento de ETV durante la gestación debe continuarse durante todo el embarazo y por lo menos seis semanas después del parto con una duración mínima de tres meses ${ }^{(49)}$.

\section{REFERENCIAS}

1. Sociedad Española de Ginecología y Obstetricia (SEGO). Enfermedad tromboembólica venosa en la gestación. Prog Obstet Ginecol. 2013;56:41-64.

2. Heit JA, Spencer FA, White RH. The epidemiology of venous thromboembolism. J Thromb Thrombolysis. 2016;41:3-14.

3. Simcox LE, Ormesher L, Tower C, Greer IA. Pulmonary thromboembolism in pregnancy: diagnosis and management. Breathe (Sheff). 2015;11:282-289.

4. Gader AA, Haggaz AE, Adam I. Epidemiology of deep venous thrombosis during pregnancy and puerperium in Sudanese women. Vasc Health Risk Manag. 2009;5:85-87.

5. Villani M, Ageno W, Grandone E, Dentali F. The prevention and treatment of venous thromboembolism in pregnancy. Expert Rev Cardiovasc Ther. 2017;15:397-402.

6. Simpson EL, Lawrenson RA, Nightingale AL, Farmer RD. Venous thromboembolism in pregnancy and the puerperium: incidence and additional risk factors from a London perinatal database. BJOG. 2001;108:56-60.

7. Heit JA, Kobbervig CE, James AH, Petterson TM, Bailey KR, Melton $\mathrm{LJ}$ 3rd. Trends in the incidence of venous thromboembolism during pregnancy or postpartum: a 30-year population-based study. Ann Intern Med. 2005;143:697-706.

8. Galambosi PJ, Gissler M, Kaaja RJ, Ulander VM. Incidence and risk factors of venous thromboembolism during postpartum period: a population-based cohort-study. Acta Obstet Gynecol Scand. 2017;96:852-861.

9. Guía de Práctica Clínica Diagnóstico y Tratamiento de la Enfermedad Tromboembólica Venosa, México; Secretaría de Salud, 2010. Disponible en: http://www.cenetec.salud.gob.mx/descargas/gpc/ CatalogoMaestro/437_GPC_Enf_troboembolica_venosa/GER_ Enfermedad tromboembxlica_venosa.pdf

10. Paz-Janeiro JL, Guzmán-Cruz LA, Pérez-Damián V, Barrios-Cedrún IR, Cortina-Nascimento A, Benites-Palacio S y cols. Manejo de la enfermedad tromboembólica venosa. Rev Mex Angiol. 2013;41:69-77.

11. Alkema L, Chou D, Hogan D, Zhang S, Moller AB, Gemmill A, et al. Global, regional, and national levels and trends in maternal mortality between 1990 and 2015, with scenario-based projections to 2030: a systematic analysis by the UN Maternal Mortality Estimation InterAgency Group. Lancet. 2016;387:462-474. 
12. Khan KS, Wojdyla D, Say L, Gülmezoglu AM, Van Look PF. WHO analysis of causes of maternal death: a systematic review. Lancet. 2006;367:1066-1074.

13. Chan LY, Tam WH, Lau TK. Venous thromboembolism in pregnant Chinese women. Obstet Gynecol. 2001;98:471-475.

14. Heit JA, Kobbervig CE, James AH, Petterson TM, Bailey KR, Melton LJ 3rd. Trends in the incidence of venous thromboembolism during pregnancy or postpartum: a 30-year population-based study. Ann Intern Med. 2005;143:697-706.

15. Velasco-Murillo V, Navarrete-Hernández E. Maternal mortality at the Mexican Institute of Social Security, from 1991 to 2005. A period of changes. Rev Med Inst Mex Seguro Soc. 2006;44:S121-128.

16. Brenner B. Haemostatic changes in pregnancy. Thromb Res. 2004;114:409-414.

17. Malavasi LO, Arêas MM. Venous thromboembolism prophylaxis in pregnancy. J Vasc Bras. 2016;15:293-301.

18. Kaski JC, Arrebola-Moreno AL. Inflamación y trombosis en la fibrilación auricular. Rev Esp Cardiol. 2011;64:551-553.

19. Urban CF, Ermert D, Schmid M, Abu-Abed U, Goosmann C, Nacken $\mathrm{W}$, et al. Neutrophil extracellular traps contain calprotectin, a cytosolic protein complex involved in host defense against Candida albicans. PLoS Pathog. 2009;5:e1000639.

20. Fuchs TA, Brill A, Wagner DD. Neutrophil extracellular trap (NET) impact on deep vein thrombosis. Arterioscler Thromb Vasc Biol. 2012;32:1777-1783.

21. Fuchs TA, Brill A, Duerschmied D, Schatzberg D, Monestier M, Myers DD Jr, et al. Extracellular DNA traps promote thrombosis. Proc Natl Acad Sci USA. 2010;107:15880-1585.

22. James AH, Tapson VF, Goldhaber SZ. Thrombosis during pregnancy and the postpartum period. Am J Obstet Gynecol. 2005;193:216-219.

23. James AH. Venous thromboembolism in pregnancy. Arterioscler Thromb Vasc Biol. 2009;29:326-331.

24. James AH, Tapson VF, Goldhaber SZ. Thrombosis during pregnancy and the postpartum period. Am J Obstet Gynecol. 2005;193:216-219.

25. Robertson L, Wu O, Greer I. Thrombophilia and adverse pregnancy outcome. Curr Opin Obstet Gynecol. 2004;16:453-458.

26. Liu S, Rouleau J, Joseph KS, Sauve R, Liston RM, Young D, et al. Epidemiology of pregnancy-associated venous thromboembolism: a population-based study in Canada. J Obstet Gynaecol Can. 2009;31:611-620.

27. De Stefano V, Martinelli I, Rossi E, Battaglioli T, Za T, Mannuccio MP, et al. The risk of recurrent venous thromboembolism in pregnancy and puerperium without antithrombotic prophylaxis. Br J Haematol. 2006;135:386-391.

28. Dilley A, Austin H, El-Jamil M, Hooper WC, Barnhart E, Evatt BL, et al. Genetic factors associated with thrombosis in pregnancy in a United States population. Am J Obstet Gynecol. 2000;183:1271-1277.

29. Robertson L, Wu O, Langhorne P, Twaddle S, Clark P, Lowe GD, et al. Thrombophilia in pregnancy: a systematic review. $\mathrm{Br}$ J Haematol. 2006;132:171-196.

30. Liston F, Davies GA. Thromboembolism in the obese pregnant woman Semin Perinatol. 2011;35:330-334.

31. Morgan ES, Wilson E, Watkins T, Gao F, Hunt BJ. Maternal obesity and venous thromboembolism. Int J Obstet Anesth. 2012;21:253-263.

32. Larsen TB, Sørensen HT, Gislum M, Johnsen SP. Maternal smoking, obesity, and risk of venous thromboembolism during pregnancy and the puerperium: a population-based nested case-control study. Thromb Res. 2007;120:505-509.

33. James AH, Jamison MG, Brancazio LR, Myers ER. Venous thromboembolism during pregnancy and the postpartum period: incidence, risk factors, and mortality. Am J Obstet Gynecol. 2006;194:1311-1315.

34. Won HS, Kim DY, Yang MS, Lee SJ, Shin H, Park JB. Pregnancy-induced hypertension, but not gestational diabetes mellitus, is a risk factor for venous thromboembolism in pregnancy. Korean Circ J. 2011;41:23-27.

35. Virkus RA, Løkkegaard E, Lidegaard Ø, Langhoff-Roos J, Nielsen AK, Rothman KJ, Bergholt T. Risk factors for venous thromboembolism in 1.3 million pregnancies: a nationwide prospective cohort. PLoS One. 2014;9:e96495.

36. Abbasi N, Balayla J, Laporta DP, Kezouh A, Abenhaim HA. Trends, risk factors and mortality among women with venous thromboembolism during labour and delivery: a population-based study of 8 million births. Arch Gynecol Obstet. 2014;289:275-284.

37. Goldhaber SZ. Epidemiology of pulmonary embolism. Semin Vasc Med. 2001;1:139-146.

38. Leung AN, Bull TM, Jaeschke R, Lockwood CJ, Boiselle PM, Hurwitz LM, et al. An official American Thoracic Society/Society of Thoracic Radiology clinical practice guideline: evaluation of suspected pulmonary embolism in pregnancy. Am J Respir Crit Care Med. 2011;184:1200-1208.

39. Damodaram M, Kaladindi M, Luckit J, Yoong W. D-dimer does notexclude venous thromboembolism (VTE) in pregnancy. J obstet Gynaecol. 2008;28:222-223.

40. Calvillo Batlles P. Algoritmo para el diagnóstico y el seguimiento de la tromboembolia pulmonar aguda. Radiología. 2017;59:75-87.

41. Greer IA. Prevention of venous thromboembolism in pregnancy. Eur J Med Res. 2004;9:135-145.

42. Okoroh EM, Azonobi IC, Grosse SD, Grant AM, Atrash HK, James AH. Prevention of venous thromboembolism in pregnancy: a review of guidelines, 2000-2011. J Womens Health (Larchmt). 2012;21:611-615.

43. Middeldorp S. How I treat pregnancy-related venous thromboembolism. Blood. 2011;118:5394-5400.

44. Greer IA, Nelson-Piercy C. Low-molecular-weight heparins for thromboprophylaxis and treatment of venous thromboembolism in pregnancy: a systematic review of safety and efficacy. Blood. 2005;106:401-407.

45. Tooher R, Gates S, Dowswell T, Davis LJ. Prophylaxis for venous thromboembolic disease in pregnancy and the early postnatal period. Cochrane Database Syst Rev. 2010;CD001689.

46. Bain E, Wilson A, Tooher R, Gates S, Davis LJ, Middleton P. Prophylaxis for venous thromboembolic disease in pregnancy and the early postnatal period. Cochrane Database Syst Rev. 2014;CD001689.

47. Carrillo ER, Saucedo AH, Núñez BJ, Contreras CN, Escárpita BN, Leal GP y cols. ¿Hospitales enfermos o pacientes enfermos? Parte II Profilaxis de la enfermedad tromboembólica venosa en la Fundación Clínica Médica Sur. Med Sur. 2007;14:176-184.

48. Chan WS, Rey E, Kent NE; VTE in Pregnancy Guideline Working Group, Chan WS, Kent NE, et al. Venous thromboembolism and antithrombotic therapy in pregnancy. J Obstet Gynaecol Can. 2014;36:527-553.

49. Bates SM, Middeldorp S, Rodger M, James AH, Greer I. Guidance for the treatment and prevention of obstetric-associated venous thromboembolism. J Thromb Thrombolysis. 2016;41:92-128. 